Meta

Journal des traducteurs

Translators' Journal

\title{
Le traducteur, le médecin et le patient
}

\section{Christian Balliu}

Volume 55, numéro 1, mars 2010

Le parcours du sens : d'une langue à l'autre — Mélanges offerts à André Clas

The Way of Meaning: From a Language to Another - Collection of Articles Offered to André Clas

URI : https://id.erudit.org/iderudit/039598ar

DOI : https://doi.org/10.7202/039598ar

Aller au sommaire du numéro

\section{Éditeur(s)}

Les Presses de l'Université de Montréal

ISSN

0026-0452 (imprimé)

1492-1421 (numérique)

Découvrir la revue

Citer cet article

Balliu, C. (2010). Le traducteur, le médecin et le patient. Meta, 55(1), 15-22.

https://doi.org/10.7202/039598ar

\section{Résumé de l'article}

Le texte médical est d'apparence scientifique et semble se caractériser par le recours aux termes. En réalité, la subjectivité y est omniprésente et se traduit par le recours aux mots, qui sont les véritables enjeux de la traduction. La terminologie ne pose pas de problème particulier au traducteur, toute l'information étant disponible grâce aux grandes banques de données documentaires, au multimédia en général et aux réseaux d'informateurs. Ce sont les mots qui véhiculent le vrai sens du texte et qui constituent un véritable défi pour le traducteur. Les termes, quant à eux, sont des leurres scientifiques qui cachent des objectifs qui le sont moins, comme la quête d'une reconnaissance scientifique, la course aux crédits de recherche ou le positionnement d'un laboratoire. D'autre part, le caractère impersonnel d'un texte médical spécialisé, qui en garantirait le caractère objectif, est de plus en plus contestable, à cause de l'importance qu'ont pris l'immunologie et la psychiatrie dans la médecine contemporaine. Ces dernières décennies,

l'immunologie et la psychiatrie ont mis en avant une conception du patient qui prend en compte le psychologique dans l'analyse des troubles physiologiques. De la sorte, le discours médical réhabilite aussi le sujet à la première personne, même si sa présence reste latente dans le texte.
Ce document est protégé par la loi sur le droit d'auteur. L’utilisation des services d'Érudit (y compris la reproduction) est assujettie à sa politique d'utilisation que vous pouvez consulter en ligne.

https://apropos.erudit.org/fr/usagers/politique-dutilisation/ 


\title{
Le traducteur, le médecin et le patient
}

\author{
CHRISTIAN BALLIU \\ Haute École de Bruxelles (ISTI), Bruxelles, Belgique \\ Institut de management et de communication interculturels (ISIT), Paris, France \\ cballiu@heb.be
}

\begin{abstract}
RÉSUMÉ
Le texte médical est d'apparence scientifique et semble se caractériser par le recours aux termes. En réalité, la subjectivité y est omniprésente et se traduit par le recours aux mots, qui sont les véritables enjeux de la traduction. La terminologie ne pose pas de problème particulier au traducteur, toute l'information étant disponible grâce aux grandes banques de données documentaires, au multimédia en général et aux réseaux d'informateurs. Ce sont les mots qui véhiculent le vrai sens du texte et qui constituent un véritable défi pour le traducteur. Les termes, quant à eux, sont des leurres scientifiques qui cachent des objectifs qui le sont moins, comme la quête d'une reconnaissance scientifique, la course aux crédits de recherche ou le positionnement d'un laboratoire. D'autre part, le caractère impersonnel d'un texte médical spécialisé, qui en garantirait le caractère objectif, est de plus en plus contestable, à cause de l'importance qu'ont pris l'immunologie et la psychiatrie dans la médecine contemporaine. Ces dernières décennies, l'immunologie et la psychiatrie ont mis en avant une conception du patient qui prend en compte le psychologique dans l'analyse des troubles physiologiques. De la sorte, le discours médical réhabilite aussi le sujet à la première personne, même si sa présence reste latente dans le texte.
\end{abstract}

\begin{abstract}
A medical text has a scientific aspect, apparently characterized by the use of terms. But in fact, there is subjectivity throughout, expressed through words which are the real issue of translation. The terminology poses no particular problem for the translator since this information is available from major documentation databases, from multimedia and from informational networks. The words convey the text's true meaning and represent the real challenge for the translator. The terms are but scientific decoys, hiding lesser targets that include the quest for scientific recognition, the race for research credits or the positioning of a laboratory. On the other hand, the impersonal character of a specialized medical text, which used to guarantee its objectivity, is now increasingly questioned because of the importance in contemporary medicine of immunology and psychiatry. In recent decades, immunology and psychiatry have highlighted a conception of the patient that takes the psychological dimension into account in the study of physiological troubles. In this way, medical discourse brings back into favour the first person subject, however discreet his presence may be in the text.
\end{abstract}

\section{MOTS-CLÉS/KEYWORDS}

médecine, sociolecte, termes, connotation, subjectivité

medicine, sociolect, terms, connotation, subjectivity

Pour André Clas, grand serviteur de la traduction

Le texte qu'on va lire n'est pas à proprement parler scientifique. Il rassemble plutôt les réflexions d'un traducteur qui en découd avec les textes médicaux depuis plus d'un quart de siècle, que ce soit sur le plan de la traduction professionnelle, de son 
enseignement, ou tout simplement de la réflexion en matière de langage médical. Il s'agit donc de livrer quelques réflexions, éprouvées par le temps et l'expérience, sur ce qu'est un texte de médecine, sur la manière dont il est écrit et sur la manière de le traduire.

Une première réflexion s'impose. Si le nombre de langues de départ peut être important, malgré la domination évidente de la langue anglaise, et si mes langues de travail sont essentiellement l'espagnol, le néerlandais et le russe, ce problème est secondaire. On ne rédigera pas la traduction en fonction d'une langue de départ particulière; le texte traduit fonctionne en toute autonomie. Si le texte traduit pouvait varier selon la langue de départ, on entrerait dès lors dans une vision linguistique de l'opération traduisante, caractérisée par un retour au comparatisme où pointerait en filigrane le fantôme du juxtalinéaire. L'idée inconsciente qui se cache derrière cette conception du juxtalinéaire est que la traduction (le produit) se juge exclusivement dans un rapport de concordance à la séquence verbale et syntaxique du texte de départ. Retrouver les mots de l'original est ce qui importera au correcteur et tout écart par rapport à l'ordre intangible du texte à traduire sera sanctionné comme un détournement lexical, syntaxique ou stylistique. Un héritage très lourd à porter et qui remonte à la Septuaginte.

À accréditer cette thèse, toujours vivace chez certains enseignants de traduction, on en viendrait à postuler qu'il n'y a qu'une version possible d'un texte, ce qui sacraliserait non seulement l'original, mais aussi sa traduction. Dans ce cas, plus de retraductions possibles, plus de facteurs sociolinguistiques en jeu, plus de dimension diachronique à prendre en compte. En un mot, l'histoire de la traduction, si précieuse dans son rôle de mise en perspective de la théorie et de la pratique, n'aurait plus aucune raison d'être dans les cursus de traduction. Souvent d'ailleurs, lorsqu'elle est présente, elle occupe une place minime et est considérée comme un simple apport culturel (ce qu'elle est aussi), alors qu'elle est un angle d'attaque particulièrement intéressant pour la réflexion traductologique.

Si le texte de départ est figé, la traduction, quant à elle, reste un possible, toujours à explorer et à reconstruire. Il n'y pas de version définitive d'un texte, canonique, à l'image de ce que devint la Vulgate à l'occasion du concile de Trente (1546-1548). Une traduction définitive scellerait le destin même de l'activité, condamnant toute tentative ultérieure et se condamnant elle-même en se momifiant dans un passé de plus en plus lointain. Ironie du sort, même sur le plan purement linguistique, la traduction deviendrait avec le temps illisible.

Ce qu'il convient de prendre en compte, c'est que l'on traduit généralement en direct dans les domaines spécialisés, a fortiori dans les domaines récents comme l'informatique ou caractérisés par une évolution rapide des connaissances et de leurs dénominations. Les deux vont souvent de pair. Les traductions littéraire et religieuse peuvent faire exception, d'où les collections des Classiques que l'on retrouve dans les grandes maisons d'édition.

En ce qui concerne la traduction médicale, la problématique est plus complexe. Si l'on assiste depuis la Seconde Guerre mondiale à une accélération des connaissances et à une floraison des techniques médicales, qu'elles soient exploratoires, diagnostiques ou thérapeutiques, la médecine est une science déjà ancienne puisqu'elle est exercée à ce titre depuis le $\mathrm{v}^{\mathrm{e}}$ siècle avant Jésus-Christ. Sa naissance est plus ancienne encore; on en trouve des traces dans les sociétés assyro-babyloniennes de l'Antiquité, 
mais elle y était pratiquée de manière incantatoire et divinatoire, à consonance exclusivement religieuse.

On peut donc aujourd'hui traduire des auteurs anciens, comme Hippocrate, Galien, Avicenne, Rhazès, Constantin l'Africain, Vésale et bien d'autres, ce qui réhabilite l'axe diachronique et sociolinguistique. Le plus souvent, on traduit bien entendu des textes contemporains, en synchronie parfaite, ce qui engendre le risque d'éluder la dimension diachronique et sociolinguistique au profit de la seule considération linguistique. Une erreur dramatique et pleine de conséquences. Le fait d'être confronté dans un nombre important de cas à une seule et même langue de départ, l'anglais, a tendance à mécaniciser la traduction, à l'aseptiser, d'autant plus que le texte original est souvent rédigé par un auteur dont la langue maternelle n'est pas l'anglais. Le traducteur a dès lors affaire à un pseudo-auteur auto-traduit. Ce seul élément montre combien une vision linguistique de l'opération traduisante en quête de correspondances avec le texte de départ n'a que peu de sens.

La dimension diachronique est donc essentielle dans l'appréhension des textes de médecine, même très récents, et est le signe qu'il convient de parler de langage médical plutôt que de langue de la médecine. Le langage médical est un sociolecte qui, au sein d'une même langue, évolue particulièrement rapidement et peut même varier en fonction des régions où il est utilisé. Ainsi, pour donner un exemple concret, un patient d'âge moyen peut désorienter complètement un jeune médecin en lui parlant d'examens subis quelques années auparavant ou en lui livrant un diagnostic asséné par un confrère il y a une décennie. L'évolution constante des dénominations recèle aussi une mise à jour, voire une correction, des notions médicales que l'on croyait établies. On pense inévitablement au jugement sans appel de Proust:

Car la médecine étant un compendium d'erreurs successives et contradictoires des médecins, en appelant à soi les meilleurs d'entre eux on a grande chance d'imposer une vérité qui sera reconnue fausse quelques années plus tard. De sorte que croire à la médecine serait la suprême folie, si n'y pas croire n'en était pas une plus grande, car de cet amoncellement d'erreurs se sont dégagées à la longue quelques vérités (Proust 1921/1988: 402).

Les dénominations s'adaptent en conséquence aux découvertes nouvelles et imposent un souci d'actualisation constante, tant aux praticiens qu'aux chercheurs, aux professeurs de faculté ou, pour ce qui est de notre objet, aux traducteurs médicaux. L'actualisation n'est pas la solution aux problèmes d'évolution du langage médical; elle n'est qu'un premier pas, indispensable certes. Il faudrait aussi que l'enseignement de l'art médical réserve une place à la variabilité des notions et des dénominations dans le temps, en d'autres termes qu'elle réhabilite le patient dans ses préoccupations. En effet, l'anamnèse est livrée par le patient, sur la base de son passé médical, lequel est aussi le produit du logos de médecins l'ayant côtoyé dans le passé. Dans l'enseignement de la médecine ainsi que dans celui de la traduction médicale, l'histoire de la discipline devrait toujours être présente en toile de fond.

Se pose dès lors le problème de la terminologie médicale dans son ensemble, particulièrement labile et mouvante, et à laquelle, malheureusement, le traducteur médical a tendance à confiner sa réflexion. Les dictionnaires et encyclopédies figent les connaissances, les rivent à un moment donné et n'envisagent que peu la notion de progrès médical, qui précisément marque une rupture par rapport à un état antérieur des connaissances et induit la création de termes nouveaux, plus appropriés aux 
découvertes récentes. Dictionnaires et encyclopédies sont des instantanés, des clichés du savoir pris sur le vif, et font l'impasse sur la consolidation progressive des savoirs dans le temps et, donc, sur la caducité des postulats scientifiques.

Le caractère scientifique, indissociable de la démarche médicale, induit l'idée d'une terminologie par essence monosémique et univoque. Pas de place pour l'ambiguïté ni pour la polysémie. En réalité, si la médecine s'efforce d'être une science, les termes qu'elle crée et véhicule ne sont pas nécessairement marqués au sceau de la monoréférentialité. Simplement parce que les termes ne renvoient pas à une réalité décharnée, déshumanisée, mais en fin de compte à l'histoire d'un individu qui se cache derrière une réalité organique, biologique ou clinique. La clinique est d'ailleurs la porte d'entrée du langage descriptif, symptomatologique, qui met en jeu le ressenti le plus profond du patient. La séméiologie, qui se dévoile notamment par l'anamnèse, est la voie ouverte au discours, tenu en l'espèce par un patient qui n'est pas spécialiste du domaine et qui met des mots sur son malaise. C'est le spécialiste qui traduit les mots en termes, un peu comme si l'utilisation de ces derniers suffisait à cautionner la véracité du propos. De même, la dénomination cryptique d'une affection confère au médecin un apparat scientifique et entretient chez le malade la confusion entre symptôme et diagnostic. En voici deux exemples simples:

(1) - Docteur, j'ai toujours le visage rougeâtre.

- Vous souffrez d'un érythème facial.

(2) - Docteur, j'ai des vertiges chaque fois que je me lève.

- Vous souffrez d'hypotension orthostatique.

Ce procédé existe depuis longtemps, on le trouve remarquablement mis en scène dans le Malade imaginaire:

ARGAN - Les médecins ne savent donc rien, à votre compte?

BERALDE - Si fait, mon frère. Ils savent la plupart de fort belles humanités, savent parler en beau latin, savent nommer en grec toutes les maladies, les définir et les diviser; mais, pour ce qui est de les guérir, c'est ce qu'ils ne savent point du tout.

ARGAN - Mais toujours faut-il demeurer d'accord que sur cette matière les médecins en savent plus que les autres.

BERALDE - Ils savent, mon frère, ce que je vous ai dit, qui ne guérit pas grand-chose, et toute l'excellence de leur art consiste en un pompeux galimatias, en un spécieux babil, qui vous donne des mots pour des raisons et des promesses pour des effets.

(Molière 1673: Acte III, Scène III)

Les termes ne sont par conséquent pas toujours les portes d'entrée qui conduisent aux notions, ils les cachent parfois ou assoient la supériorité cognitive du médecin par rapport au patient, auquel l'accès à la connaissance est volontairement entravé par l'opacité du discours. Cette opacité peut aussi compenser l'impuissance du praticien sur le terrain, lequel se donne une aura scientifique qui rassure le patient sur son efficacité thérapeutique. C'est le cas de l'expression abstention thérapeutique, d'usage si fréquent dans le discours médical et qui signifie généralement incapacité thérapeutique.

Si les termes doivent être par définition aseptisés, objectifs, neutres et décrire des réalités observables, vérifiables, scientifiques, ils ne constituent que les briques de l'appareil discursif, en aucune manière le liant. C'est alors que les mots, les expressions, 
les métaphores viennent en quelque sorte au secours des termes en réhabilitant le domaine dont il est fondamentalement question, c'est-à-dire l'homme et, à travers lui, la vie tout simplement, avec ce que la quête éperdue de la santé, la course - perdue d'avance - contre la mort peut avoir de subjectif, d'affectif, d'angoissant. Le traducteur médical doit autant s'enquérir des mots que des termes.

La connotation du texte médical est cependant difficile à accepter en raison d'un néopositivisme qui bannit l'affectif de la démarche purement intellectuelle, alors que celle-ci s'appuie sur une part importante d'intuition. Le hasard de certaines grandes découvertes médicales n'est qu'une coïncidence apparente; en réalité, on ne trouve souvent que ce que l'on cherche.

Ainsi, à y regarder de près, le discours médical contemporain est beaucoup plus connoté que l'on ne pourrait le penser en première analyse, y compris dans des textes très spécialisés. Ce discours traduit une double rupture. D’abord, par rapport à une médecine organique qui considérait l'homme comme une constellation d'organes et la santé comme un équilibre essentiellement physiologique. Ensuite, par rapport à une médecine générale (ne parle-t-on pas de médecin généraliste?) qui s'est émiettée, voire effritée, dès 1950 en un foisonnement de domaines de plus en plus spécialisés et donc de plus en plus étroits. En considérant les choses avec un peu de recul, on pourrait dire que l'intégralité nosologique du patient s'était réduite à des monades organiques; le général avait cédé la place au particulier.

Deux éléments viennent expliquer, en partie du moins, l'irruption de la connotation dans les textes contemporains de médecine spécialisée. D’une part, la naissance de l'immunologie comme discipline médicale à part entière dans les années 1960. Jusqu'alors, l'immunologie était abordée de manière ponctuelle dans les études de médecine, constituait presque un excursus qui apparaissait secondaire en regard de l'organicité du propos. L'immunologie, c'est l'idée qu'il existe un individu global.

Les deux mots sont importants: le patient est à la fois unique et forme par ailleurs une entité homogène, indissociable, ce qu'occulte précisément l'approche organique. En voici un exemple entendu lors d'une consultation pour un trouble hépatique:

- Docteur, j’ai aussi des migraines et des angoisses.

- Nous verrons cela plus tard! Je m'occupe d'abord de votre foie.

Un deuxième exemple, tout aussi significatif:

Il est regrettable que le développement de cette magnifique tumeur ait été interrompu par le décès du patient.

Enfin, un dernier exemple mettant en scène un chirurgien attendant son patient en salle d'opération:

- Montez le foie du quatrième [étage].

Cette relégation au second plan du psychologique sur l'autel du physiologique évacue à tort la dimension psychosomatique, qu'aucun médecin actuel n'oserait cependant explicitement dénier dans son discours. Elle signe aussi la récupération de la maladie par le médecin.

L'immunologie traverse horizontalement la structuration organiciste en restaurant une vision holistique de la médecine, qui considère le système immunitaire comme un ensemble homogène et organisé de défense de l'organisme contre des 
corps étrangers. Le ton est donné dans le vocabulaire utilisé; les mots défense et étrangers qui véhiculent l'idée de lutte contre un ennemi dotent l'individu d'une conscience de soi.

C'est précisément la distinction entre le soi et le non-soi (le trait d'union est essentiel) qui est à la base de l'immunologie et qui fut formulée par Frank Burnet ${ }^{1}$ (Self and Notself) en 1969. On voit poindre en creux le phénomène du rejet (le mot est tout aussi significatif), qui fut éprouvé lors de la première transplantation cardiaque effectuée la même année par le professeur Christian Barnard.

L'immunologie, c'est donc la prise de conscience du rôle actif de l'individu dans le combat contre les agents infectieux. Et cette prise de conscience a transformé le sociolecte médical en lui conférant une tournure résolument active et un lexique de plus en plus militaire pour traduire le combat mené contre les affections. Le mot patient retrouve de la sorte son sens premier.

Les syntagmes batterie de tests, cohorte de patients, arsenal thérapeutique, mécanismes de défense, autant d'expressions à connotation militaire qui symbolisent la lutte sans merci contre la maladie. Plus la lutte est inégale, plus l'ennemi est difficile à combattre, plus le vocabulaire employé deviendra agressif, parfois pour masquer l'incapacité curative.

Le deuxième élément qui a favorisé le recours à la métaphore dans le discours médical est le développement de la psychiatrie et de la psychanalyse en Europe occidentale ces dernières décennies. La psychiatrie a mis en évidence le concours du psychisme dans la nosogenèse, c'est-à-dire la naissance des maladies. On sait que la dépression ou la tristesse peuvent jouer un rôle dans l'apparition de certains cancers ou que les anesthésistes ont tendance à s'inquiéter des patients nerveux ou angoissés par l'intervention chirurgicale.

La psychiatrie, en remettant en selle l'importance du psychologique dans la préservation de la santé ou dans la survenue de la maladie, a également redonné toute sa puissance au logos comme facteur d'identification, et d'amélioration dans le cadre d'une psychothérapie, de certaines affections. Le ce n'est rien, c'est nerveux est aujourd'hui discrédité dans la pratique médicale et l'on a appris que les troubles fonctionnels peuvent être les prodromes de troubles lésionnels: le rôle de l'angoisse dans l'apparition des ulcères ou de l'eczéma n'est plus à démontrer; la composante psychologique du psoriasis fait aujourd'hui l'unanimité, avec d'autres cofacteurs comme l'auto-immunité.

Parler peut soulager, voire guérir, qu'il s'agisse du discours du patient ou de celui que lui adresse le thérapeute. Le sujet pensant retrouve toute sa force et détruit la vision strictement organique du corps au profit d'une entité éminemment plus complexe qui associe un système structuré de défense de l'organisme et une participation active du sujet à ce système. Il n'est pas étonnant en conséquence que le discours médical dans son ensemble soit connoté, même si cette connotation est pernicieuse.

Cette connotation peut être militaire comme signalé plus haut. À l'inverse, le vocabulaire peut devenir complaisant et être la litote d'une réalité nosologique plus inquiétante. On parlera au patient anxieux d'un kyste au lieu d'une tumeur, d'un geste au lieu d'une intervention chirurgicale. Même s'agissant de termes, on parlera plutôt d'anémie (terme entré dans l'usage courant, mais qui désigne littéralement la privation totale de sang) que d'érythrocytopénie (déficit en globules rouges), terme 
scientifiquement exact mais qui pourrait, en raison de sa formation savante et cryptique, terrifier le patient à son annonce. De même, la néoplasie peut aussi remplacer la tumeur. Dans ce dernier exemple, on remarquera que la terminologisation (néoplasie) ou la déterminologisation (kyste) recèlent une réalité identique (tumeur).

Le traducteur doit connaître ces pratiques discursives, qui sont tantôt destinées à renforcer la position du médecin, tantôt dictées par l'élusion d'un diagnostic douloureux. Le discours médical privilégie dans sa relation au patient les tournures elliptiques et métonymiques, dans certains cas simplement par souci d'économie linguistique. Ainsi, la tumeur cervicale n'est pas un cancer du cou, mais un cancer du col de l'utérus. Le spécialiste utilise donc consciemment une dénomination incomplète et fausse, et tous ses confrères comprennent la vérité qui se cache derrière l'inexactitude.

Le traducteur s'aperçoit à la lumière de tels exemples (et ils sont très nombreux) que la terminologie médicale se pare d'une auréole scientifique, mais que dans la réalité cette terminologie savante est court-circuitée par d'autres enjeux. Ces enjeux sont parfois égocentriques, l'exercice de l'art de guérir étant aussi une lutte de pouvoir vis-à-vis du patient ou d'autres confrères. L'éponymie médicale, fréquente, dérobe au patient sa pathologie pour que le médecin qui a découvert l'agent infectieux ou la maladie (et parfois l'a guérie) puisse entrer à jamais dans l'histoire de la médecine; songeons à la maladie d'Alzheimer, au bacille de Koch, à la maladie de Parkinson. C'est tellement vrai que le nom du médecin associé à la maladie peut varier selon les pays: le goitre exophtalmique est appelé Graves disease ou Parry disease en GrandeBretagne, alors qu'en Allemagne cette même maladie s'appelle Basedow Krankheit, d'après le médecin allemand von Basedow. En Italie, la même maladie prend le nom de malattia di Flajani, en hommage au médecin italien Giuseppe Flajani. En France, pays qui n'a pas revendiqué de paternité en la matière, l'affection porte le nom de maladie de Basedow (Kernbaum 2008: 123).

En y réfléchissant bien, on se rend compte que la connotation relaie dans les textes les enjeux profonds de la médecine, qui sont notamment la course aux crédits de recherche, à la notoriété ${ }^{2}$, au mandarinat. Et chercher, c'est produire, donc publier, donc être lu. La diffusion des résultats de la recherche se fait par la voie des publications qui doivent être convaincantes, persuasives, assertives. La conviction et la persuasion ont recours à la connotation latente, afin de ne pas dénaturer la scientificité du propos.

Dans le discours médical, les termes sont l'arbre scientifique qui cache la forêt d'intérêts subjectifs, lesquels sont par ailleurs parfois légitimes: un laboratoire ne peut en effet vivre sans subsides extérieurs, qui permettent les progrès de la science mais qui sont octroyés sur la base de résultats probants.

C'est le subjectif que doit guetter le traducteur derrière l'apparence objective de tout texte médical, afin de traduire le véritable objet du propos. Paradoxalement, ce sont les mots qui évoquent le non-dit. Les termes ne sont qu'un problème de langue, soluble par le recours aux dictionnaires, aux encyclopédies médicales et aux bases de données documentaires. Les mots ressortissent au discours, au cœur même de l'acte de traduire. 


\section{NOTES}

1. Frank Burnet obtint le prix Nobel de médecine en 1960, pour ses travaux inédits en immunologie.

2. À ce propos, il suffit de penser à la polémique qui a entouré le récent prix Nobel de médecine 2008 accordé aux Français Luc Montagnier et Françoise Barré-Sinoussi pour la découverte du VIH, alors que le professeur Jean-Claude Chermann n’a pas été associé à ce prix.

\section{RÉFÉRENCES}

Burnet, Frank (1969) : Cellular Immunology, Self and Notself. Cambridge: Cambridge University Press.

Kernbaum, Serge, dir. (2008) : Dictionnaire de médecine Flammarion. $8^{e}$ éd. Paris: Flammarion Médecine-Sciences.

Molière, Jean-Baptiste Poquelin, dit (1965/1673): Le Malade imaginaire. Paris: Librairie Larousse.

Proust, Marcel (1921/1988) : À la recherche du temps perdu - Le Côté de Guermantes. Tome 3. Paris: France Loisirs. 